\title{
Shockley-Frank stacking faults in 6H-SiC
}

Jianwu Sun, T. Robert, A. Andreadou, A. Mantzari, Valdas Jokubavicius, Rositsa Yakimova,

J. Camassel, S. Juillaguet, E. K. Polychroniadis and Mikael Syväjärvi

\section{Linköping University Post Print}

N.B.: When citing this work, cite the original article.

Original Publication:

Jianwu Sun, T. Robert, A. Andreadou, A. Mantzari, Valdas Jokubavicius, Rositsa Yakimova, J. Camassel, S. Juillaguet, E. K. Polychroniadis and Mikael Syväjärvi, Shockley-Frank stacking faults in 6H-SiC, 2012, Journal of Applied Physics, (111), , 113527.

http://dx.doi.org/10.1063/1.4729064

Copyright: American Institute of Physics (AIP)

http://www.aip.org/

Postprint available at: Linköping University Electronic Press

http://urn.kb.se/resolve?urn=urn:nbn:se:liu:diva-78494 


\section{AIP Applied Physics}

\section{Shockley-Frank stacking faults in $6 \mathrm{H}-\mathrm{SiC}$}

J. W. Sun, T. Robert, A. Andreadou, A. Mantzari, V. Jokubavicius et al.

Citation: J. Appl. Phys. 111, 113527 (2012); doi: 10.1063/1.4729064

View online: http://dx.doi.org/10.1063/1.4729064

View Table of Contents: http://jap.aip.org/resource/1/JAPIAU/v111/i11

Published by the American Institute of Physics.

\section{Related Articles}

Effect of stacking fault and temperature on deformation behaviors of nanocrystalline $\mathrm{Mg}$ J. Appl. Phys. 112, 054322 (2012)

Atomic structure of prismatic stacking faults in nonpolar a-plane GaN epitaxial layers Appl. Phys. Lett. 101, 112102 (2012)

Importance of excitonic effects and the question of internal electric fields in stacking faults and crystal phase quantum discs: The model-case of $\mathrm{GaN}$

J. Appl. Phys. 112, 053512 (2012)

Ruddlesden-Popper faults in $\mathrm{LaNiO} 3 / \mathrm{LaAlO} 3$ superlattices

J. Appl. Phys. 112, 013509 (2012)

Optical and structural studies of homoepitaxially grown m-plane GaN

Appl. Phys. Lett. 100, 172108 (2012)

\section{Additional information on J. Appl. Phys.}

Journal Homepage: http://jap.aip.org/

Journal Information: http://jap.aip.org/about/about_the_journal

Top downloads: http://jap.aip.org/features/most_downloaded

Information for Authors: http://jap.aip.org/authors

\section{ADVERTISEMENT}

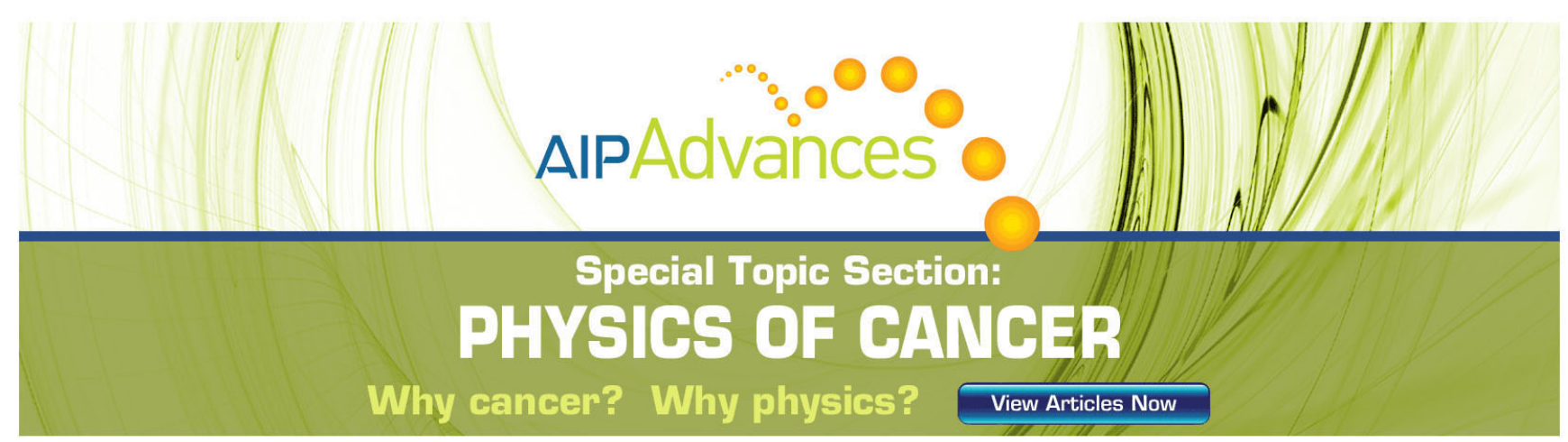




\title{
Shockley-Frank stacking faults in $6 \mathrm{H}-\mathrm{SiC}$
}

\author{
J. W. Sun, ${ }^{1,2, a)}$ T. Robert, ${ }^{3}$ A. Andreadou, ${ }^{4}$ A. Mantzari, ${ }^{4}$ V. Jokubavicius, ${ }^{1}$ R. Yakimova, ${ }^{1}$ \\ J. Camassel, ${ }^{2}$ S. Juillaguet, ${ }^{3}$ E. K. Polychroniadis, ${ }^{4}$ and M. Syväjärvi ${ }^{1}$ \\ ${ }^{1}$ Department of Physics, Chemistry and Biology, Linköping University, SE-58183 Linköping, Sweden \\ ${ }^{2}$ CNRS, Laboratoire Charles Coulomb UMR 5221, F-34095 Montpellier, France \\ ${ }^{3}$ Université Montpellier 2, Laboratoire Charles Coulomb UMR 5221, F-34095 Montpellier, France \\ ${ }^{4}$ Department of Physics, Aristotle University of Thessaloniki, GR-54124 Thessaloniki, Greece
}

(Received 8 December 2011; accepted 10 May 2012; published online 13 June 2012)

We report on Shockley-Frank stacking faults (SFs) identified in $6 \mathrm{H}-\mathrm{SiC}$ by a combination of low temperature photoluminescence (LTPL) and high resolution transmission electron microscopy (TEM). In the faulted area, stacking faults manifested as large photoluminescence emissions bands located in between the $6 \mathrm{H}-\mathrm{SiC}$ signal (at $\sim 2.99 \mathrm{eV}$ ) and the 3C-SiC bulk-like one (at $\sim 2.39 \mathrm{eV}$ ). Each of the stacking fault related emission band had a four-fold structure coming from the TA, LA, $\mathrm{TO}$, and LO phonon modes of 3C-SiC. Up to four different faults, with four different thickness of the $3 \mathrm{C}-\mathrm{SiC}$ lamella, could be observed simultaneously within the extent of the laser excitation spot. From the energy of the momentum-conservative phonons, they were associated with excitonic energy gaps at $E_{g x 1}=2.837 \mathrm{eV}, E_{g x 2}=2.689 \mathrm{eV}, E_{g x 3}=2.600 \mathrm{eV}$ and $E_{g x 4}=2.525 \mathrm{eV}$. In the same part where low temperature photoluminescence was performed, high resolution transmission electron microscopy measurements revealed stacking faults which, in terms of the Zhdanov notation, could be recognized as SFs $(3,4),(3,5),(3,6),(3,7),(3,9),(3,11),(3,16)$ and $(3,22)$, respectively. Among them stacking fault $(3,4)$ was the most common one, but a faulted region with a $(4,4) 8 \mathrm{H}-\mathrm{SiC}$ like sequence was also found. Using a type II $6 \mathrm{H} / 3 \mathrm{C} / 6 \mathrm{H}$ quantum-well model and comparing with experimental results, we find that the photoluminescence emissions with excitonic band gaps at $2.837 \mathrm{eV}\left(E_{g x 1}\right), 2.689 \mathrm{eV}\left(E_{g x 2}\right), 2.600 \mathrm{eV}\left(E_{g x 3}\right)$ and $2.525 \mathrm{eV}\left(E_{g x 4}\right)$ come from SFs $(3,4),(3,5),(3,6)$ and $(3,7)$, respectively. A possible formation mechanism of these SFs is suggested, which involves a combination of Frank faults with Shockley ones. This provides a basic understanding of stacking faults in $6 \mathrm{H}-\mathrm{SiC}$ and gives a rapid and non-destructive approach to identify SFs by low temperature photoluminescence. (C) 2012 American Institute of Physics. [http://dx.doi.org/10.1063/1.4729064]

\section{INTRODUCTION}

For many years, silicon carbide ( $\mathrm{SiC})$ has been considered as a promising material for the next generation of electronic devices. This includes the full range of high power, high frequency, and high temperature applications that are necessary in a modern industry. ${ }^{1}$ Unfortunately, despite the large development of crystal growth technologies, the full commercialization of SiC-based devices has been largely hampered by the high density of structural defects which still manifest in most available commercial wafers. During the last ten years the density of macroscopic defects like hollow core dislocations (micropipes) and small-angle boundaries has been significantly reduced. ${ }^{2-4}$ However, the presence of stacking faults (SFs) still remains a critical issue. Particularly, when off-axis substrates are used, the problem of SFs becomes extremely severe. For instance, SFs induced in bipolar devices under a strong electrical stress increase the series resistance of diodes and result in permanent device degradation. ${ }^{5}$

For a long time, this problem has slowed down the development of $\mathrm{SiC}$ bipolar devices. ${ }^{5,6}$ Different types of SFs

\footnotetext{
a) Author to whom correspondence should be addressed. Electronic mail: jianwusun@gmail.com
}

have been theoretically investigated to increase understanding of their presence and formation. In $4 \mathrm{H}-\mathrm{SiC}$, focusing on pure Shockley SFs (SSFs) introduced by the motion of partial dislocations, Iwata et al. investigated the corresponding energy levels. ${ }^{7-10}$ They showed that pure SSFs in a hexagonal $(4 \mathrm{H}$ or $6 \mathrm{H}) \mathrm{SiC}$ matrix lead to narrow bands (lamellae) of the 3C polytype which develop perpendicular to the c-axis. In this case, because of the large conduction band offset between the cubic inclusions and the hexagonal matrix, they act as perfect type II quantum wells (QWs) which can confine electrons. This triggered experimental interest and, since that time, many groups have reported on SSFs in 4H-SiC samples. ${ }^{11}$

Most of the experimental results combine low temperature photoluminescence (LTPL) spectroscopy with transmission electron microscopy (TEM). In this way, not only SSFs with a $3 \mathrm{C}$-like structure but also SFs made of $8 \mathrm{H}$-like ribbons have been identified. ${ }^{11}$ Since they exhibit different thicknesses of $3 \mathrm{C}$ or $8 \mathrm{H}-\mathrm{SiC}$ lamellas, according to the $\mathrm{QW}$ model, one should expect the observations of SF-related emissions at different energy positions. From LTPL spectroscopy, this is indeed what is found and different emission bands of SFs in $4 \mathrm{H}-\mathrm{SiC}$ have been largely reported from photoluminescence and cathodoluminescence spectroscopy. To date, these LTPL signatures provide a fast and non-destructive approach to 
evaluate the thickness of as-grown defects and to determine whether different types of SFs co-exist or not in a given $4 \mathrm{H}-\mathrm{SiC}$ material.

In $6 \mathrm{H}-\mathrm{SiC}$, the situation is totally different. From the theoretical calculations, it was predicted that a single Shockley SF, labeled as $1 \mathrm{SSF}$, had a very low formation energy of $\sim 3.6 \mathrm{~mJ} / \mathrm{m}^{2}$ and should be the most common defect. ${ }^{10}$ Unfortunately, on the experimental side there are very few works focusing on the investigation of SSFs in $6 \mathrm{H}-\mathrm{SiC} .{ }^{12,13}$ Takahashi et al. ${ }^{12}$ observed a high density of SFs in $6 \mathrm{H}-\mathrm{SiC}$ which resulted in sizeable anomalies of the resistivity. According to the QW model, they suggested that this most probably appears because the electrons were trapped in anisotropic SFs ribbons. However, despite the low formation energy expected from the theoretical predictions, they were not single Shockley SF. Instead, high-resolution TEM (HRTEM) data collected on a reasonable number of specimens revealed that SFs were produced by inserting or removing one single Si-C bilayer in the $6 \mathrm{H}$ matrix. ${ }^{12}$ Such defects constitute the so-called extrinsic or intrinsic Frank faults. In a later work, Liu et al. ${ }^{13}$ also reported different intrinsic and extrinsic Frank faults in $6 \mathrm{H}-\mathrm{SiC}$ but, again, without observation of the predicted single Shockley SF.

Opposite to the extensive series of optical and structural studies of SFs in $4 \mathrm{H}-\mathrm{SiC}$, there is no detailed investigation of the microstructure of $\mathrm{SFs}$ in $6 \mathrm{H}-\mathrm{SiC}$. As a consequence, there is no basic understanding of the radiative recombination spectrum associated with a specific SF structure and there is even no knowledge of which SF is the most common one, and whether it behaves as a QW for the electrons or not.

In this work, we focus on $6 \mathrm{H}-\mathrm{SiC}$ epitaxial layers grown on $1.4^{\circ}$ off-axis substrates. From a combination of LTPL spectroscopy with HR-TEM images collected in the same epilayer area, we evidence that different SFs co-exist in $6 \mathrm{H}-$ $\mathrm{SiC}$. None of them is the simple 1SSF defect expected from the theoretical calculations but the TEM images evidence the different (faulted) sequences. In contrast to the standard combination of single Shockley SFs usually found in $4 \mathrm{H}-$ $\mathrm{SiC}$, we find that most of the common defects can only be explained by a combination of Frank and Shockley formation mechanism. Similar with the situation in $4 \mathrm{H}-\mathrm{SiC}$, we find that a type II QW model can perfectly describe various types of SFs observed in $6 \mathrm{H}-\mathrm{SiC}$. This provides the basic understanding of SFs in $6 \mathrm{H}-\mathrm{SiC}$ and gives a rapid and nondestructive approach to check such SFs by LTPL spectra, and thus gives a quick feedback to the material growth.

\section{EXPERIMENTALS}

Thick $6 \mathrm{H}-\mathrm{SiC}$ epilayers were grown on $1.4^{\circ}$ off-axis (0001) 6H-SiC substrates (SiCrystal) by the fast sublimation growth process (FSGP). ${ }^{14}$ The typical layer thickness is around $200 \mu \mathrm{m}$ and growth time is $1 \mathrm{~h}$. The growth was performed in a high purity graphite crucible at $1775^{\circ} \mathrm{C}$. Fig. 1 shows the schematic view of the growth configuration in which a source sublimes and the resultant vapor species nucleate on the substrate. As is known, 3C-SiC can easily nucleate on an on-axis $6 \mathrm{H}-\mathrm{SiC}$ substrate while homoeptaxial step-flow growth is usually achieved on a larger off-axis $\left(3.5^{\circ}\right)$

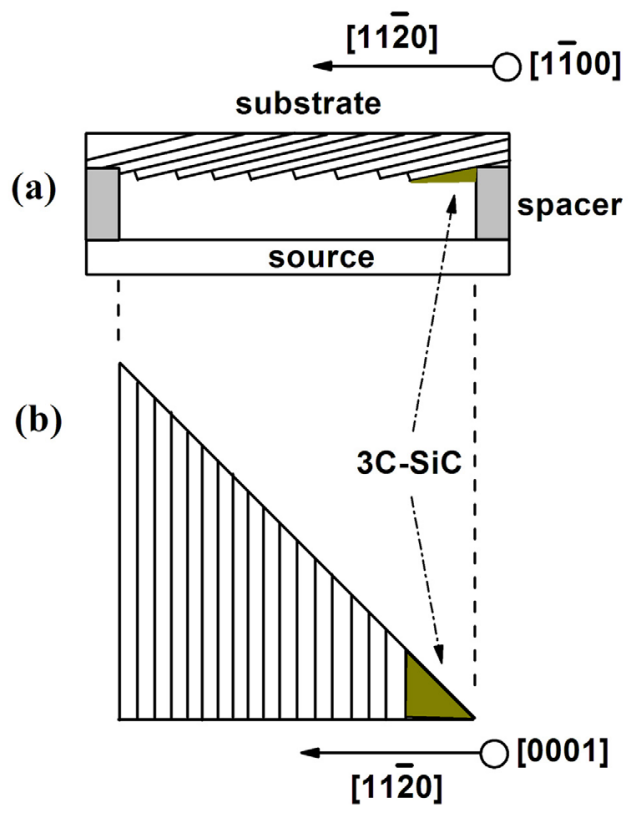

FIG. 1. (a) Schematic cross-sectional view of the growth configuration used for the sublimation epitaxial growth. (b) Top view of the epilayer through the triangular opening spacer. The shaded part shows the terrace of off-axis substrate where $3 \mathrm{C}-\mathrm{SiC}$ can easily nucleate.

substrate. On a low off-axis substrate, there is a high probability of spontaneous two-dimensional nuleation of $3 \mathrm{C}-\mathrm{SiC}$ on the terraces of the step. This is illustrated in the Fig. 1(b). Here, we used a spacer with an opening of triangular shape to minimize the area of long terraces on low off-axis substrate. A $6 \mathrm{H}-\mathrm{SiC}$ epilayer grown with such a spacer is shown by optical microscope picture in the inset of Fig. 2(d). In the corner of the triangular layer, a small area of $3 \mathrm{C}-\mathrm{SiC}$ was grown while most part was dominated by $6 \mathrm{H}-\mathrm{SiC}$ homoepitaxial growth. The detailed growth information can be found in Ref. 15 . We have also used circle-, square-shaped, and rectangular spacers for the growth on $1.4^{\circ}$ off-axis $6 \mathrm{H}-\mathrm{SiC}$ substrates and all the epilayers exhibit identical PL properties as the triangular sample when samples contain 3C-SiC. Thus, in this work, we use triangular epilayer as an example for discussion.

The LTPL spectra have been collected at $5 \mathrm{~K}$, using 30 $\mathrm{mW}$ of the $244 \mathrm{~nm}$ wavelength of a frequency doubled $\mathrm{Ar}^{+}$ion laser as excitation source. The laser spot was focused on the sample with a diameter of $\sim 100 \mu \mathrm{m}$. A Triax spectrometer from Horiba-Jobin Yvon fitted with a $600 \mathrm{~g} / \mathrm{mm}$ gratings and a cooled CCD camera completed the set-up.

The TEM specimens were prepared in cross-sectional orientation first by mechanical grinding to a thickness of approximately $30 \mu \mathrm{m}$. Then, the thinning process continued by $\mathrm{Ar}^{+}$ ion milling until electron transparency. The observations were performed on a JEOL 2011 HRTEM working at $200 \mathrm{kV}$.

\section{RESULTS AND DISCUSSION}

\section{A. LTPL spectra of stacking faults}

The LTPL spectra from different locations (points A, B, $\mathrm{C}$, and $\mathrm{D}$ ) of a $6 \mathrm{H}-\mathrm{SiC}$ triangular layer are shown in Fig. 2. Point $\mathrm{A}$ is close to the edge of the $6 \mathrm{H}-\mathrm{SiC}$ area and the point $\mathrm{D}$ is at the corner of the triangular layer in the $3 \mathrm{C}-\mathrm{SiC}$ area. 


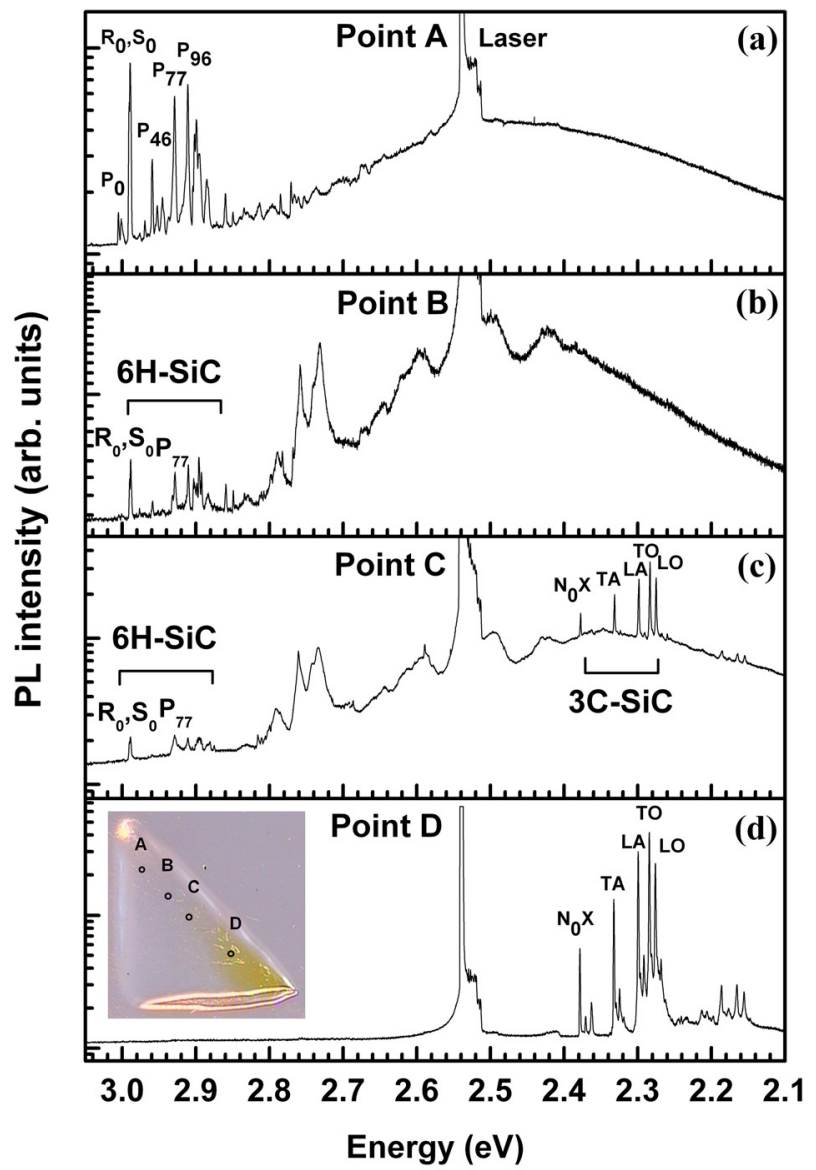

FIG. 2. LTPL spectra collected at $5 \mathrm{~K}$ on different locations of a $6 \mathrm{H}-\mathrm{SiC}$ layer, as indicated in the inset of Fig. 2(d). The circles A, B, C, and D denote the locations where the LTPL measurements were performed on the sample.

Points $\mathrm{B}$ and $\mathrm{C}$ are located at positions stepping from the $6 \mathrm{H}-\mathrm{SiC}$ area toward the $3 \mathrm{C}-\mathrm{SiC}$ area. The locations are shown in the inset of Fig. 2(d). In the $6 \mathrm{H}-\mathrm{SiC}$ matrix area near point $\mathrm{A}$, the PL spectrum is typical of low doped n-type $6 \mathrm{H}-\mathrm{SiC}$. It shows the characteristics of the radiative recombination lines of nitrogen bound excitons, with corresponding phonon replicas, but no signal coming from $3 \mathrm{C}-\mathrm{SiC}$. Three zero-phonon lines $\left(\mathrm{P}_{0}, \mathrm{R}_{0}\right.$, and $\left.\mathrm{S}_{0}\right)$ of $\mathrm{N}$ bound exciton emis- sions can be observed clearly. These are associated with the recombination of a bound exciton at the hexagonal and two cubic sites in $6 \mathrm{H}-\mathrm{SiC}$, respectively. In addition to these $\mathrm{N}$ bound excitons and phonon replicas, a broad band centered at $\sim 2.45 \mathrm{eV}$ is also observed. Compared to the typical N-Al donor-acceptor-pair (DAP) emission in $6 \mathrm{H}-\mathrm{SiC}$, this new band is much broader and shows a large shift of about $0.19 \mathrm{eV}$ lower than the maximum of the N-Al DAP emission band. ${ }^{16}$ Thus, this emission band does not originate from N-Al DAP emission. More probably it comes from unidentified defects rather than specific doping species.

Moving to point $\mathrm{B}$ in the $6 \mathrm{H}-\mathrm{SiC}$ matrix area, the PL intensities of $\mathrm{N}$ bound excitons and phonon replicas are significantly decreased while a series of new LTPL features manifest in this broad band. Each of them is made of fourfold structures and quite similar to the well-known LTPL signatures of $3 \mathrm{C}-\mathrm{SFs}$ in $4 \mathrm{H}-\mathrm{SiC} .{ }^{11}$ In the following, we will demonstrate that these new series of features are indeed indicative of a specific stacking fault. Also at this point, there is no $\mathrm{PL}$ signal from $3 \mathrm{C}-\mathrm{SiC}$. When moving to point $\mathrm{C}$, the PL spectrum shows very weak $\mathrm{N}$ bound excitons and phonon replicas from $6 \mathrm{H}-\mathrm{SiC}$ but stronger near band-edge emissions from 3C-SiC, namely, $\mathrm{N}$ bound exciton $\left(\mathrm{N}_{0} \mathrm{X}\right)$ and its four phonon replicas (TA, TO, LA, LO). ${ }^{17}$ More importantly, all of the series of SFs signatures which appear in between the $6 \mathrm{H}-\mathrm{SiC}$ matrix signals and the $3 \mathrm{C}-\mathrm{SiC}$ lines are exactly same as those observed in point B. Finally, the area of point $\mathrm{D}$ was only governed by near-band-edge emissions from 3C-SiC without any contributions from $6 \mathrm{H}-\mathrm{SiC}$ or SFs. This clearly indicates that all the SFs from observed PL spectrum exist in the $6 \mathrm{H}-\mathrm{SiC}$ matrix but not $3 \mathrm{C}-\mathrm{SiC}$.

It should be noted that all the observed PL features are not specific in this triangular epilayer. Actually, in all the epilayers grown with different shape spacers (circle-, squareshaped, triangular, and rectangular), we observed the same LTPL spectra trends from $6 \mathrm{H}-\mathrm{SiC}$ area to $3 \mathrm{C}-\mathrm{SiC}$ part and, especially, identical stacking faults emissions as those observed in the triangular epilayer.

To further illustrate PL signatures for stacking faults, a part of the LTPL spectrum collected at point $C$ is plotted in Fig. 3. We recognize easily the near band-edge emissions

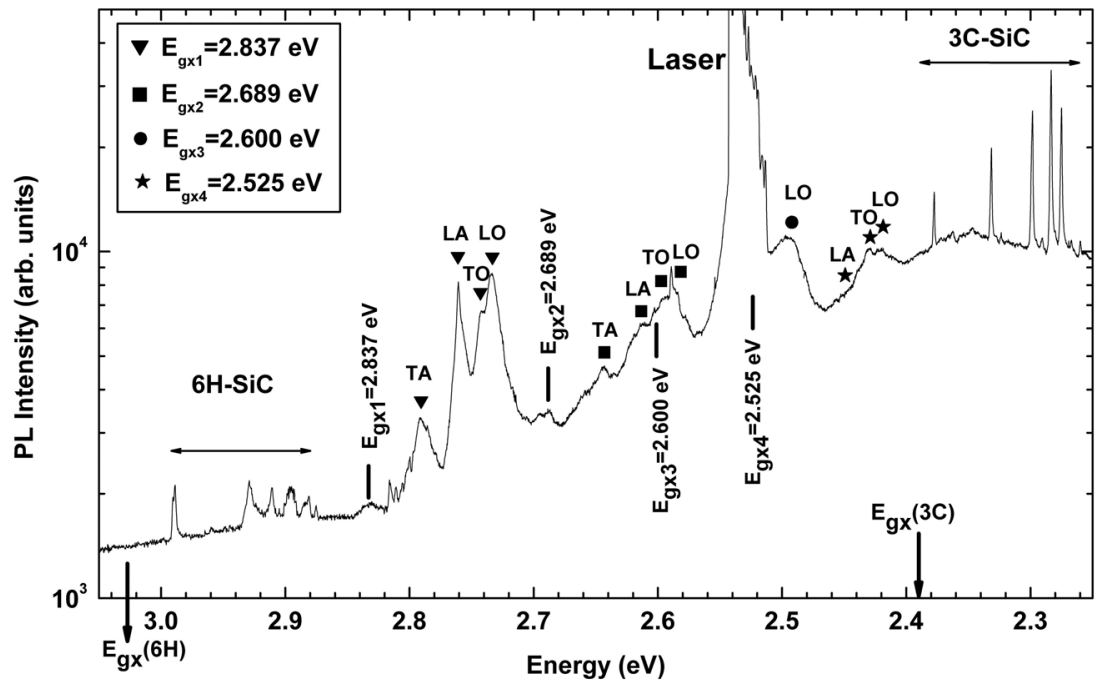

FIG. 3. Part of LTPL spectrum collected from point C shown in Fig. 2(c). A series of large, similar, QW-like structures which appear in between the $6 \mathrm{H}-\mathrm{SiC}$ matrix signal (at $\sim 2.99 \mathrm{eV}$ ) and the bulk-like 3C-SiC lines (at $\sim 2.39 \mathrm{eV}$ ) are the specific SFs signatures (showing by triangles, squares, dots, and stars) and all series of broad features are indicative of a specific SF lamella. 
( $\mathrm{N}$ bound excitons and phonon replicas) in the large $6 \mathrm{H}$ and $3 \mathrm{C}-\mathrm{SiC}$ parts of crystal. They manifest near $2.99 \mathrm{eV}$ for $6 \mathrm{H}-\mathrm{SiC}$ and $2.38 \mathrm{eV}$ for $3 \mathrm{C}-\mathrm{SiC}$, respectively, as narrow lines. The four series of features which manifest in between these near band-edge emissions are broad and each of them has a fourfold component structure.

Four well-resolved components denoted by triangles are located at $2.791,2.761,2.742$, and $2.734 \mathrm{eV}$, respectively. Their energy separations correspond to the TA (46 meV), LA (76.6 meV), TO (94.5 meV), and LO (104.5 meV) phonon replicas associated with $3 \mathrm{C}-\mathrm{SiC}$ and are considered as typical optical signatures of SFs. ${ }^{11}$ Following the same observation of phonon separations, we can identify four SFs simultaneously as shown in Fig. 3 with some of their phonon replicas being superimposed with the laser line. From the energies of momentum-conserving phonons, we find the excitonic band gaps $E_{g x}$ at 2.837, 2.689, 2.600, and $2.525 \mathrm{eV}$ for SFs indicated by triangles, squares, dots, and stars, respectively.

To summarize this part, we demonstrate that from the LTPL spectra the epilayer shown in the inset of Fig. 2(d) converts from $6 \mathrm{H}$ - to $3 \mathrm{C}-\mathrm{SiC}$ when moving from point $\mathrm{A}$ to D. Only in the $6 \mathrm{H}-\mathrm{SiC}$ matrix, four kinds of SFs can be clearly resolved and they have a LTPL signature similar to those of the SFs previously reported in $4 \mathrm{H}-\mathrm{SiC}$ in which they are recognized by a type II QW model. ${ }^{11}$ Assuming the same basic features, since the excitonic energy gap in Fig. 3 are much higher than the band gap energy of 3C-SiC, we expect the thickness of the SF lamellae to be very thin (and the quantum confinement to be the dominant effect). We shall come back on this point in Sec. V.

\section{B. HR-TEM images of stacking faults}

High-resolution TEM was carried out in the part of the epilayer near points B and C where the SFs were observed from LTPL spectra. In Figs. 4 to 6, the cross-sectional HRTEM images show (at least) 10 different kinds of SFs in this area which are different from the results of theoretical calculations. From ab-initio super-cell calculations, Iwata et al. ${ }^{10}$ predicted only one to four Shockley SFs, namely $1 \mathrm{SSF}(4,2)$, $2 \operatorname{SSF}(5,1), 3 \operatorname{SSF}(9,3)$ and $4 \operatorname{SSF}(10,2)$ in the Zhdanov notation. They found that the $1 \mathrm{SSF}(4,2)$ has the lowest formation energy of about $3.6 \mathrm{~mJ} / \mathrm{m}^{2}$ which is about six to seven times smaller than that of second stacking fault $2 \mathrm{SSF}(5,1)$. However, we did not observe such a SF with sequence $(4,2)$ or $(2,4)$ in spite of its lowest formation energy by calculation. The only predicted SF which can be observed from TEM is $3 \mathrm{SSF}(9,3)$ containing three successive glides in the formation mechanism (namely, 3 Shockley SFs). This is shown in Fig. 4(b), where we show part of the inverse fast Fourier transformed (IFFT) processed image taken from Fig. 4(a). Clearly, one can see a fault sequence $(3,9)$, which is essentially same as $3 \operatorname{SSF}(9,3)$ if the observer counts the sequence from the other side of the crystal. However, no other predicted SFs can be observed from TEM images. This seriously questions the Shockley formation mechanism.

In the faulted area, the most often observed fault in HR-TEM is SFs $(3,4)$, as seen in Table I. As an example, Fig. 4(c) shows SFs $(3,4)$ embedded in $6 \mathrm{H}-\mathrm{SiC}$ units. More-

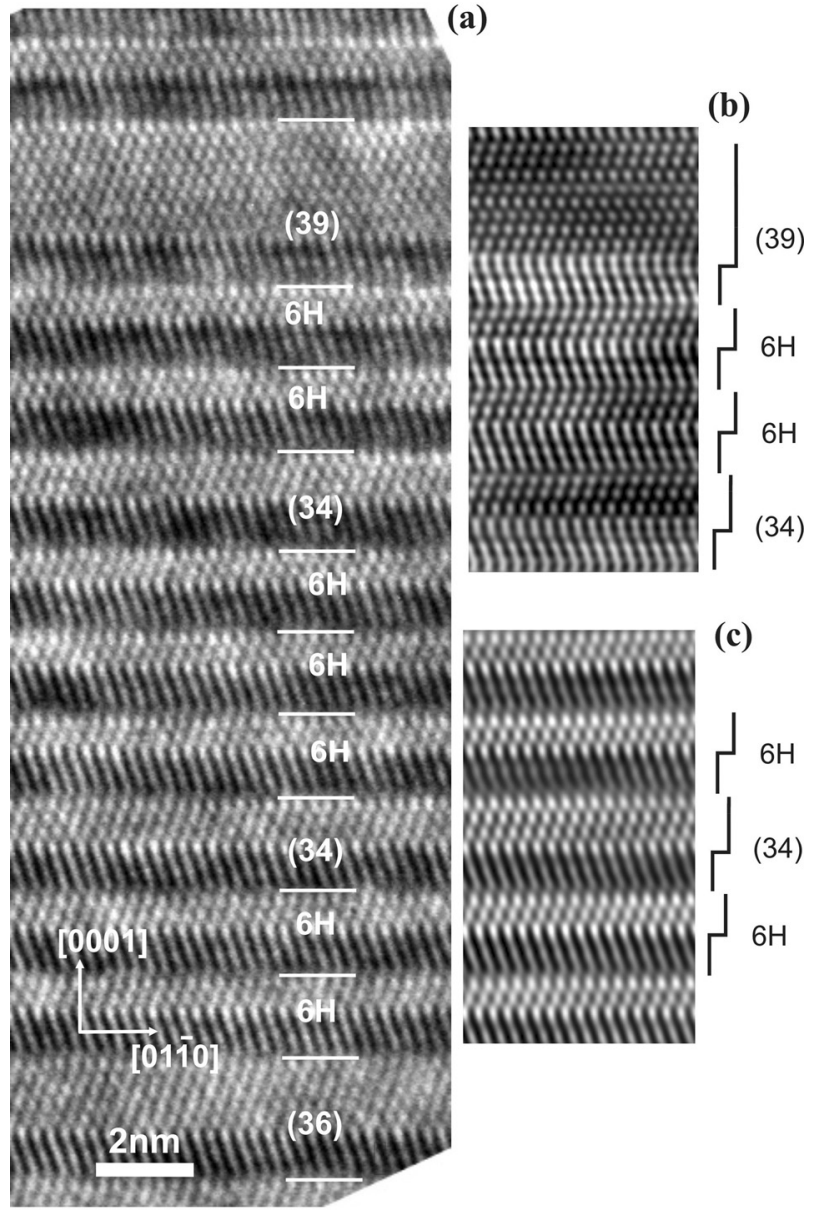

FIG. 4. (a) Cross-sectional HR-TEM image collected from the area near points $\mathrm{B}$ and $\mathrm{C}$ of the sample shown in the inset of Fig. 2(d), (b) IFFT processed image from part of the image (a), showing SFs $(3,9)$, (c) IFFT processed image from part of the image (a), showing SFs $(3,4)$.

over, an $8 \mathrm{H}$-like faulted lamella $(4,4)$ was also observed in Fig. 5. Apart from SFs $(3,4)$ and $(4,4)$, several stacking faults in sequences of $(3,5),(3,6),(3,7)$ can also be observed. At the bottom of Fig. 5(a), the faulted area manifests a series of successive SFs $(3,4),(3,6),(3,7)$, and $(3,5)$. As it is well known, the SFs in $4 \mathrm{H}-\mathrm{SiC}$ can be recognized as either $3 \mathrm{C}$ or $8 \mathrm{H}$ ribbons and, at least in the case of $3 \mathrm{C}$, exhibit different thicknesses. ${ }^{11}$ However, for the SFs in $6 \mathrm{H}-\mathrm{SiC}$ discussed above, it is rather difficult to identify the 3C-SiC lamella and to define the thickness and we will come back to this point in Sec. IV. As seen in Fig. 6, SFs $(3,11),(3,16),(3,22)$ contain thicker 3C-SiC lamellas. In Table I, we summarize all the SFs observed from HR-TEM images. The number for each SF indicates how many times it appeared in the TEM measurements. Similarly, from HR-TEM images, Liu et al. found the SFs in $6 \mathrm{H}-\mathrm{SiC}$ with the faulted sequences of $(3,2),(3,4)$, $(3,5),(3,6)$, and $(3,2,2,2),{ }^{13}$ which are also including in Table I. This indicates that all the SFs we observed are not specific in this $6 \mathrm{H}-\mathrm{SiC}$ sample.

\section{FORMATION MECHANISMS OF STACKING FAULTS}

A common formation mechanism of stacking faults in $4 \mathrm{H}$ or $6 \mathrm{H}-\mathrm{SiC}$ is the so-called Shockley SF mechanism. In this case, a SF is introduced by a partial dislocation glide 


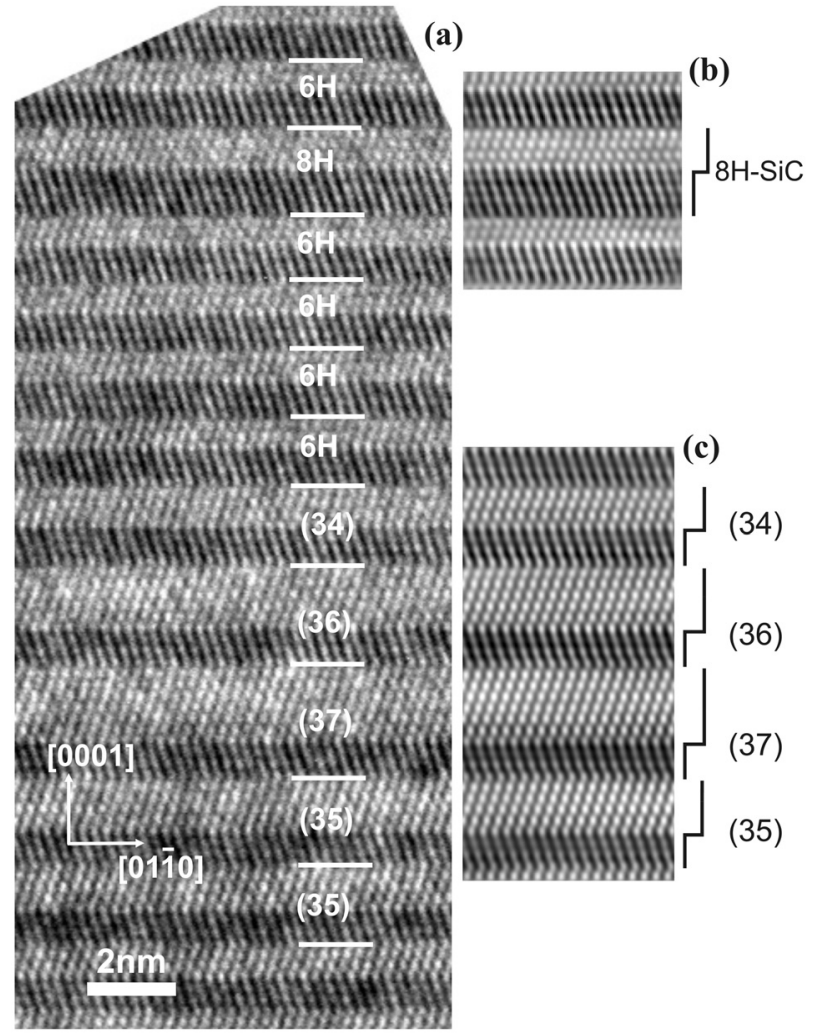

FIG. 5. (a) Cross-sectional HR-TEM image collected from the area near points $B$ and $C$ of the sample shown in the inset of Fig. 2(d), (b) IFFT processed image from part of the image (a), showing $8 \mathrm{H}$-like SFs $(4,4)$, (c) IFFT processed image from part of the image (a), showing an arrange of successive SFs $(3,4),(3,6),(3,7),(3,5)$.
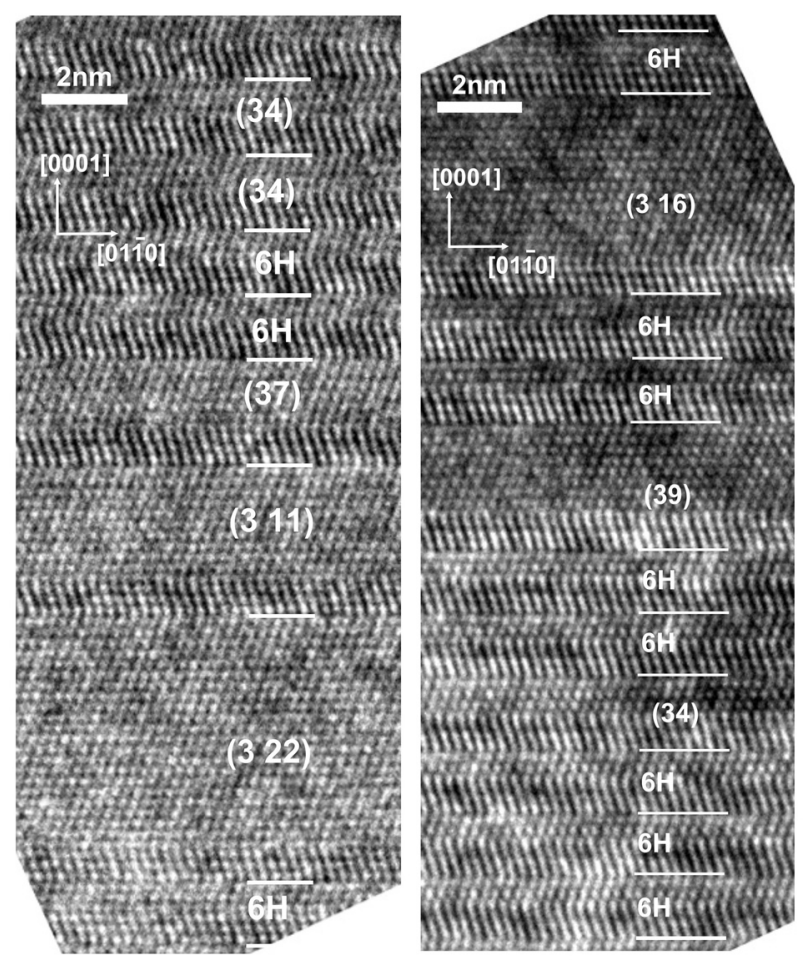

FIG. 6. Cross-sectional HR-TEM image collected from the area near points $\mathrm{B}$ and $\mathrm{C}$ of the sample shown in the inset of Fig. 2(d), in which the stacking faults containing the larger $3 \mathrm{C}$ lamellas were also observed [labeled as $(3,11),(3,16),(3,22)]$. in the basal (0001) plane, where part of the crystal above the glide plane is rigidly shifted with relative to the lower part. ${ }^{11}$ First, we consider the formation of $\operatorname{SF}(3,9)$ by simple Shockley glide mechanism. In the Hägg notation, the stacking sequence of perfect $6 \mathrm{H}-\mathrm{SiC}$ is expressed as:

$$
\begin{aligned}
& \ldots(+++---)(+++---)(+++---) \\
& \quad(+++---) \ldots
\end{aligned}
$$

Simply introducing three glide planes by the propagation of partial dislocations, the stacking sequence will change to

$$
\begin{aligned}
& \ldots(+++---)(+++---) \mid(-|-|----) \\
& \quad(+++---) \ldots,
\end{aligned}
$$

where the glide plane was indicated as "l". While this fault formation mechanism may explain convincingly the formation of the $3 \operatorname{SSF}(3,9)$ defects in Figs. 4 and 6, it is rather difficult to use it to form the other SFs. This is simply because the total number of bilayers in the faulted sequence is no longer a multiple of three. This is clearly seen in Fig. 4(c) where the stacking sequence of $\mathrm{SFs}(3,4)$ embedded in the "correct" $6 \mathrm{H}$ sequence $(3,3)$ cannot be created by a glide. We need then to consider the second type of SFs formation mechanism which calls for the coalescence of point defects, either vacancies or interstitials. This is the so-called Frank SF formation mechanism. The coalescence of vacancies removes a part of hexagonal layer and results in an intrinsic frank SF (IFSF). On the opposite, inserting an additional layer (because of the coalescence of interstitial atoms) gives rise to an extrinsic frank SF (EFSF).

To explain the formation mechanisms for the observed SFs, we suggest a model which is illustrated in terms of $\mathrm{ABC}$ and Zhdanov notations, as seen in Fig. 7. Starting from the stacking sequence of the perfect $6 \mathrm{H}-\mathrm{SiC}$, one can easily produce SFs $(3,4)$ by inserting an additional bilayer and gliding one bilayer, as shown in Fig. 7(c). This means the faults $(3,4)$, which is observed several times from TEM images, can be formed by a combination of one EFSF with one SSF. As seen, Fig. 7(c) shows the $\operatorname{SFs}(3,4)$ embedded in the correct $6 \mathrm{H}$ sequence $(3,3)$, which is exactly same as that found from TEM image in Fig. 4(c). It should be noted that SFs $(3,4)$ have one more quasicubic bilayers with respect to perfect $6 \mathrm{H}-\mathrm{SiC}(3,3)$, seen Figs. 7(a) and 7(c). Similarly, as shown in Fig. 7(d), two EFSFs can simply create a faulted lamella with $8 \mathrm{H}$-like stacking sequence $(4,4)$ embedded in $6 \mathrm{H}-\mathrm{SiC}$ matrix. Furthermore, if one bilayer of 2EFSFs was shifted in the basal plane and results in one additional Shockley SFs (see Fig. $7(\mathrm{e})$, the layer 10 was shifted), the net result is the formation of SFs(3,5). By a comparison of Figs. 7(a) and 7(e), $\mathrm{SFs}(3,5)$ have two more quasi-cubic bilayers with respect to $6 \mathrm{H}-\mathrm{SiC}(3,3)$. If one continues to shift three successive bilayers above $\operatorname{SFs}(3,5)$, namely layers 15,16 , and 17 in Fig. 7 (e), the SFs $(3,11)$ can be formed. In the same way, three EFSFs can create the $\operatorname{SFs}(3,6)$ and four EFSFs accompanied with $1 \mathrm{SSF}$ can give rise to $\operatorname{SFs}(3,7)$. For the larger SFs $(3,16)$ and $(3,22)$, the formation mechanism can 
TABLE I. Different types of stacking faults observed from HR-TEM measurements near points B and C in sample shown in the inset of Fig. 2(d). The number of each specific SF was counted from HR-TEM images. For comparison, all the SFs observed in Ref. 13 are also included.

\begin{tabular}{|c|c|c|c|c|c|c|c|c|c|c|c|}
\hline SFs & $(3,4)$ & $(3,5)$ & $(3,6)$ & $(3,7)$ & $(3,9)$ & $(3,11)$ & $(3,16)$ & $(3,22)$ & $(3,2)$ & $(4,4)$ & $(3,2,2,2)$ \\
\hline Numbers observed in this work & 9 & 2 & 3 & 3 & 1 & 1 & 1 & 1 & 1 & 1 & 0 \\
\hline $\mathrm{E}_{\mathrm{gx}}$ from LTPL $(\mathrm{eV})$ & 2.837 & 2.689 & 2.6 & 2.525 & $\ldots$ & $\ldots$ & $\ldots$ & $\ldots$ & $\ldots$ & $\ldots$ & $\ldots$ \\
\hline QW width (BLs) & 1 & 2 & 3 & 4 & $\ldots$ & $\ldots$ & $\ldots$ & $\ldots$ & $\ldots$ & $\ldots$ & $\ldots$ \\
\hline Observed in Ref. 13 & Yes & Yes & Yes & $\ldots$ & $\ldots$ & $\ldots$ & $\ldots$ & $\ldots$ & Yes & $\ldots$ & Yes \\
\hline
\end{tabular}

also be explained in the same way by a multiple combination of Shockley SFs and extrinsic Frank SFs.

To summarize, all the stacking faults observed from TEM results can be explained by inserting one or more bilayers and sometimes accompanied with glides (Shockley SFs). More importantly, by a comparison of the stacking sequence of these faults and perfect $6 \mathrm{H}-\mathrm{SiC}$, it is found that SFs $(3,4)$ have one more quasi-cubic bilayers and SFs $(3,5)$ have two more quasi-cubic bilayers with respect to the perfect $6 \mathrm{H}-\mathrm{SiC}(3,3)$ and in the same way for SFs $(3,6),(3,7)$ and so on. We also observed a faulted ribbon with sequence of $(3,2)$ embedded in 6H-SiC (TEM image is not shown here). This can be explained by a combination of one Shockley SF and one intrinsic Frank SFs (removal of one bilayer from perfect $6 \mathrm{H}-\mathrm{SiC}$ ).

Of course, if the total number of bilayers for the faulted area is a multiple of three, there is also another possibility to form the faults only by applying glides in the basal plane. This means by using only Shockley SFs. As an example, we consider the faulted region shown at the bottom of Fig. 5(a), which shows a series of successive SFs in the stacking sequence of $(3,3),(3,4),(3,6),(3,7)$, $(3,5),(3,5)$, and $(3,3)$. There are 54 bilayers in total and, in the Hägg notation, the stacking sequence of the nine successive $6 \mathrm{H}-\mathrm{SiC}$ unit cells which correspond to 54 successive $\mathrm{Si}-\mathrm{C}$ bilayers, is simply:

$$
\begin{gathered}
\ldots(+++---)(+++---)(+++---) \\
(+++---)(+++---)(+++---) \\
(+++---)(+++---)(+++---) \ldots
\end{gathered}
$$

By applying 20 glides (means 20 Shockley SFs), one can get a faulted lamella with the sequence given below:

$$
\begin{aligned}
& \ldots(+++---)(+++---)|(-++\mid+--)| \\
& \quad(-|-|--|+|+)(+|-|----)|(-|-+|+\mid+-)| \\
& (-|-|--|+|+)(+|-|----)(+++---) \ldots,
\end{aligned}
$$

which is same as the series of successive SFs $(3,3),(3,4),(3,6)$, $(3,7),(3,5),(3,5)$, and $(3,3)$ observed in the bottom of Fig. 5(a). However, this formation mechanism seems too complicated and not so realistic because too many glides are needed.

\section{CALCULATION BY QUANTUM-WELL MODEL AND COMPARISON WITH EXPERIMENTAL RESULTS}

We have observed at least four kinds of SFs from LTPL spectra and in the part of the sample where LTPL was performed, we also found several types of SFs with different stacking sequences from HR-TEM. In order to know which kind of SF can contribute to a specific PL emission, one needs to know the electronic structures of these SFs.

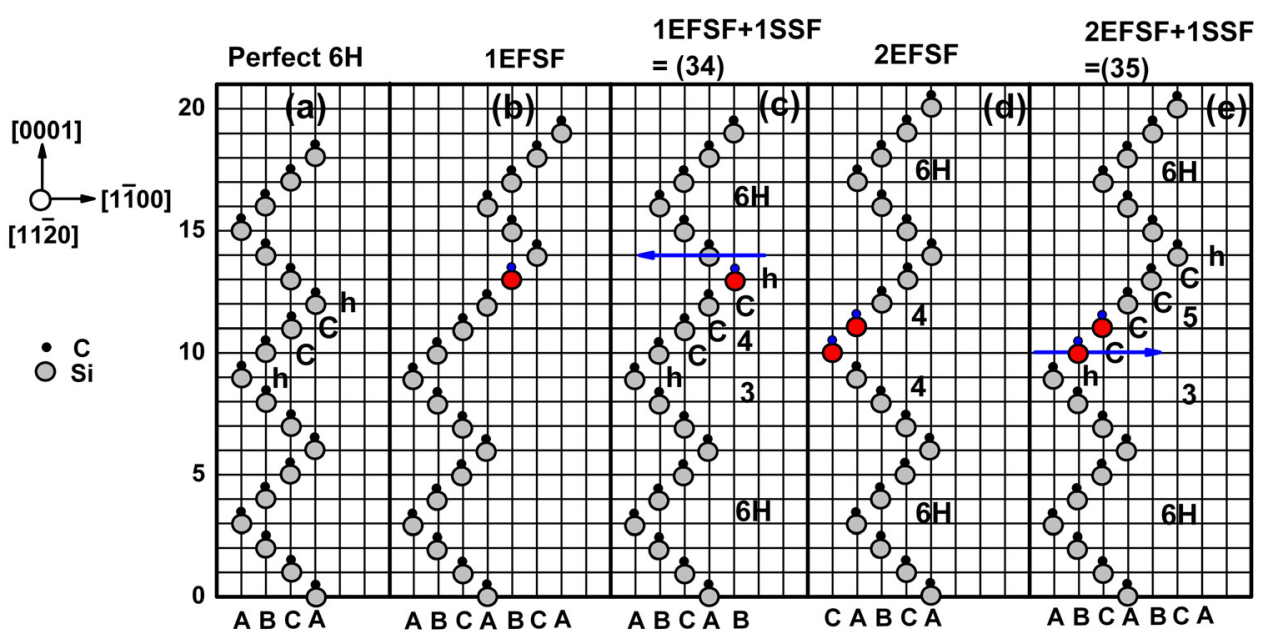

FIG. 7. Schematic diagram of the stacking faults in $6 \mathrm{H}-\mathrm{SiC}$ viewed along the [11 20$]$ direction. Stacking sequences are illustrated in terms of the ABC and Zhdanov notations. The red symbols represent the inserting bilayers and the blue arrow denotes the glide plane. (a) perfect $6 \mathrm{H}-\mathrm{SiC}$; (b) one extrinsic Frank SF formed by inserting one additional bilayer; (c) SFs $(3,4)$ obtained by combining one extrinsic Frank SF with one Shockley SF; (c) 8H-like SFs (4, 4) obtained by two extrinsic Frank SF; (d) SFs $(3,5)$ formed by two extrinsic Frank SF and one Shockley SF. The signs "h" or "c" denote Si-C bilayers that reside in quasi-hexagonal or quasi-cubic environments. Note that SFs $(3,4)$ have one more quasi-cubic bilayers and SFs $(3,5)$ have two more quasi-cubic bilayers, with respect to the perfect $6 \mathrm{H}-\mathrm{SiC}(3,3)$. 
It has been established by the theoretical calculations that strongly localized wave functions associated with a SF have most of their amplitude in the region the stacking sequence is more $3 \mathrm{C}$-like. ${ }^{7}$ It was found that the conduction band minimum in $3 \mathrm{C}-\mathrm{SiC}$ is well below the conduction band minimum in $4 \mathrm{H}-$ or $6 \mathrm{H}-\mathrm{SiC}(\sim 1 \mathrm{eV}$ below in $4 \mathrm{H}$ and $\sim 0.64 \mathrm{eV}$ in $6 \mathrm{H}$ ), whereas the corresponding valence band offsets are much smaller. ${ }^{9}$ Therefore, if the faulted $3 \mathrm{C}$-like region is formed in $4 \mathrm{H}-$ or $6 \mathrm{H}-\mathrm{SiC}$, the conduction electrons tend to be confined in the faulted $3 \mathrm{C}$-like region according to a type II QW model. From the comparison of the supercell calculations, it is further supported that the QW interpretations is indeed realistic. Especially for SFs in $4 \mathrm{H}-\mathrm{SiC}$, the experimental results both coming from the PL and TEM can be explained very well according to the type II $4 \mathrm{H} / 3 \mathrm{C} / 4 \mathrm{H}$ QW model. ${ }^{11}$ Thus we follow the same way as used in $4 \mathrm{H}-\mathrm{SiC}$ to explain the SFs observed in $6 \mathrm{H}-\mathrm{SiC}$.

In the inset of Fig. 8, we present a schematic drawing of a type II QW structure for a $3 \mathrm{C}-\mathrm{SiC}$ lamella in a $6 \mathrm{H}-\mathrm{SiC}$ matrix. The corresponding band structure parameters used for QW calculations are listed in Table II. ${ }^{9,18}$ Apart from the band offsets, there are two major factors which determine the recombination energy in a $\mathrm{QW}$, namely quantum confinement and Stark effect due to the in-built electronic field. In hexagonal $\mathrm{SiC}$, the four tetrahedral $\mathrm{C}$-Si bonds are not equivalent and bond-to-bond charge transfer and ionic relaxation can cause an intrinsic spontaneous polarization directed opposite to the c-axis in crystal. ${ }^{11}$ Since spontaneous polarization does not exist in 3C-SiC for symmetry, the electric dipole moment per unit volume should be a maximum in $2 \mathrm{H}-\mathrm{SiC}$ and proportional to the degree of hexagonality in the other polytypes $(100 \%$ in $2 \mathrm{H}-, 50 \%$ in $4 \mathrm{H}-$ and $33 \%$ in $6 \mathrm{H}-$ $\mathrm{SiC})$. As a result, the electric field in $6 \mathrm{H} / 3 \mathrm{C} / 6 \mathrm{H} \mathrm{QW}$ can be estimated to be $0.8 \mathrm{MV} / \mathrm{cm}$, which is scaled down from the obtained value of $1.2 \mathrm{MV} / \mathrm{cm}$ for $4 \mathrm{H} / 3 \mathrm{C} / 4 \mathrm{H}$ QW (after

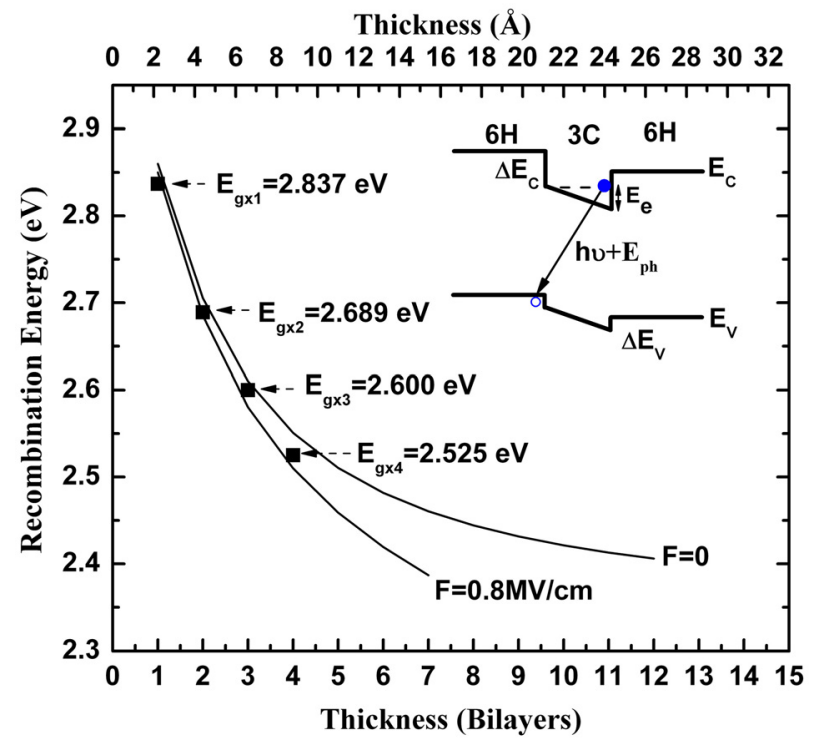

FIG. 8. Calculated recombination energies in a type II QW structure $6 \mathrm{H} / 3 \mathrm{C} /$ $6 \mathrm{H}$ using TMM technique as a function of the width of QW. Black squares represent the experimental recombination energies obtained from LTPL spectrum in Fig. 3. The inset shows the schematic drawing of a type II QW structure for a $3 \mathrm{C}-\mathrm{SiC}$ lamella in a $6 \mathrm{H}-\mathrm{SiC}$ matrix.
TABLE II. Band structure parameters used in this work to perform the calculation of a type II QW model. The conduction band offset $\Delta E_{C}$ is give by $E_{C}(6 \mathrm{H})-E_{C}(3 \mathrm{C})$ and valence band offset $\Delta E_{V}=E_{V}(6 \mathrm{H})-E_{V}(3 \mathrm{C})$.

\begin{tabular}{lcc}
\hline \hline & $6 \mathrm{H}-\mathrm{SiC}$ & $3 \mathrm{C}-\mathrm{SiC}$ \\
\hline$E_{g}(\mathrm{eV})$ & 3.0 & 2.39 \\
$m_{e}\left(m_{0}\right)$ & 1.25 & 0.316 \\
$\Delta E_{C}(\mathrm{meV})$ & 650 & 0 \\
$\Delta E_{V}(\mathrm{meV})$ & 40 & 0 \\
\hline \hline
\end{tabular}

Ref. 11) according to the percentage of hexagonality. By using a constant of the electric field, the QW can be described as a triangular well and the optical emission energy $h v$ is expressed by ${ }^{11}$

$$
h v=E_{g x}(3 C)+E_{e}-e F L-\Delta E_{V}-E_{p h},
$$

where $E_{e}$ is the ground state energy of an electron in the well, $L$ is the width of the well, and $E_{p h}$ is the energy of the momentum-conserving phonon. Thus, the effective excitonic band gap of the $\mathrm{QW}$ is given by $E_{g x}(\mathrm{QW})=h v+E_{p h}$.

To calculate the recombination energy as a function of the width of the QW, we numerically solve the Schrödinger equation for the QW with different widths by a transfer matrix method (TMM) which is described in Ref. 11 . The calculated results are shown in Fig. 8 for a type II $6 \mathrm{H} / 3 \mathrm{C} / 6 \mathrm{H}$ QW with and without electric field. We also plot the experimental recombination energies of SFs derived from LTPL spectra, namely, $E_{g x 1}=2.837, E_{g x 2}=2.689, E_{g x 3}=2.600$, and $E_{g x 4}$ $=2.525 \mathrm{eV}$. They can fit the calculated curve very well if one assumes that the thickness of quantum well (3C lamella) is from one bilayer to four bilayers for them, respectively. In fact, in the Sec. IV, we demonstrate that $\operatorname{SFs}(3,4), \operatorname{SFs}(3,5)$, $\operatorname{SFs}(3,6)$, and $\mathrm{SFs}(3,7)$ have one to four more quasi-cubic CSi bilayers with respect to perfect $6 \mathrm{H}-\mathrm{SiC}(3,3)$, respectively.

As seen in Figs. 7(a) and 7(c), the perfect $6 \mathrm{H}-\mathrm{SiC}$ has a periodic sequence of one quasi-hexagonal " $h$ " site and two quasi-cubic "c" sites while the $\operatorname{SFs}(3,4)$ follows the exact same periodic sequence except one additional quasi-cubic bilayer. This suggests that $\operatorname{SFs}(3,4)$ can be understood by inserting one cubic-like bilayer in the perfect $6 \mathrm{H}-\mathrm{SiC}$. It seems to be reasonable because compared to the perfect $6 \mathrm{H}$ $\mathrm{SiC}$, the only difference of periodic crystal potential felt by an electron in $\mathrm{SFs}(3,4)$ comes from this additional quasicubic bilayer. Similarly, $\mathrm{SFs}(3,5)$ have two more quasi-cubic bilayers with respect to the perfect $6 \mathrm{H}-\mathrm{SiC}(3,3)$, as seen in Fig. 7(e). In the same way, $\operatorname{SFs}(3,6)$ can be understood by inserting three quasi-cubic bilayers in a perfect $6 \mathrm{H}-\mathrm{SiC}$ and SFs $(3,7)$ is nothing but four more additional quasi-cubic bilayers in $6 \mathrm{H}-\mathrm{SiC}$. Consequently, SFs $(3,4),(3,5),(3,6)$, and $(3,7)$ can be regarded as $6 \mathrm{H} / 3 \mathrm{C} / 6 \mathrm{H}$ QWs with the well width of one, two, three, and four cubic bilayers, respectively, for the calculation shown in Fig. 8. Hence, the strong PL emissions with the $E_{g x 1}=2.837 \mathrm{eV}$ most probably come from SFs $(3,4)$. An experimental evidence is given by the fact that SFs $(3,4)$ is the most common observed SFs in TEM images while the strongest PL emissions from LTPL spectra are associated with $E_{g x 1}=2.837 \mathrm{eV}$. Then, we are led to 
conclude that $E_{g x 1}=2.837, E_{g x 2}=2.689, E_{g x 3}=2.600$, and $E_{g x 4}=2.525 \mathrm{eV}$ are related to SFs $(3,4)$, SFs $(3,5)$, SFs $(3,6)$, and SFs $(3,7)$, respectively, as shown in Table I.

In Fig. 8, the experimental recombination energies can fit both the curves calculated with and without electric field in $6 \mathrm{H} / 3 \mathrm{C} / 6 \mathrm{H} \mathrm{QWs}$. This is because that for such thinner QWs, the quantum confinement effect is dominated and in-built electric field does not cause a large redshift. For the SFs containing larger cubic inclusions such as SFs $(3,22)$, they probably behave like a bulk $3 \mathrm{C}-\mathrm{SiC}$ in the photoluminescence.

It is obvious that the observation area during a HRTEM study is very limited with respect to the illumination area (laser spot diameter $\sim 100 \mu \mathrm{m}$ ) in the PL measurements. This means that the PL spectra correspond to a lot of SFs, a certain number of which are observed and studied by TEM. These SFs are grouped into several types according to the specific sequence of the $\mathrm{Si}-\mathrm{C}$ bilayers. The PL signals which are found to correspond to specific SF types, are statistical effect, coming from these SF groups. Although the HRTEM study was performed in several neighboring area of the sample, it is difficult to count the density of each SF group and give a measure of their comparative frequency.

\section{CONCLUSION}

In conclusion, different types of SFs in $6 \mathrm{H}-\mathrm{SiC}$ were systematically investigated by a combination of LTPL and TEMimaging. Four types of SFs can be clearly indentified simultaneously within the extent of the laser excitation from LTPL measurements. The excitonic energies associated with the radiative recombinations at these SFs were found at $E_{g x 1}=2.837$, $E_{g x 2}=2.689, E_{g x 3}=2.600$, and $E_{g x 4}=2.525 \mathrm{eV}$, respectively. In the same part where LTPL was performed, HR-TEM results showed that different types of SFs can be recognized as SFs $(3,4),(3,5),(3,6),(3,7),(3,9),(3,11),(3,16)$, and $(3,22)$, respectively. Moreover, a faulted region with $8 \mathrm{H}-\mathrm{SiC}$ like sequence of $(4,4)$ was also observed by TEM in the same area. From a Schrödinger calculation of type II $6 \mathrm{H} / 3 \mathrm{C} / 6 \mathrm{H}$ QW model and comparison with experimental results, we found that the PL emissions with the excitonic band gaps at $E_{g x 1}=2.837, E_{g x 2}$ $=2.689, E_{g x 3}=2.600$, and $E_{g x 4}=2.525 \mathrm{eV}$ are related to SFs
$(3,4),(3,5),(3,6)$, and $(3,7)$, respectively. Moreover, a possible formation mechanism of these SFs was also suggested by a combination of Frank faults and Shockley faults.

\section{ACKNOWLEDGMENTS}

This work was supported by Ångpanneföreningen Research Foundation, Richerts Foundation, Swedish Energy Agency, Nordic Energy Research, Swedish Research Council (Project No. 2009-5307).

${ }^{1}$ For a recent review, see, in Silicon Carbide: Recent Major Advances, edited by W. Choyke, H. Matsunami, and G. Pensl (Springer, New York, 2004).

${ }^{2}$ J. Heindl, W. Dorsch, H. P. Strunk, St. G. Müller, R. Eckstein, D. Hofmann, and A. Winnacker, Phys. Rev. Lett. 80, 740 (1998).

${ }^{3}$ N. Ohtani, T. Fujimoto, M. Katsuno, T. Aigo, and H. Yashiro, Mater. Sci. Forum 389-393, 29 (2002).

${ }^{4}$ St. G. Müller, R. C. Glass, H. M. Hobgood, V. F. Tsvetkov, M. Brady, D. Henshall, D. Malta, R. Singh, J. Palmour, and C. H. Carter, Jr., Mater. Sci. Eng., B 80, 327 (2001).

${ }^{5}$ J. P. Bergman, H. Lendenmann, P. A. Nilsson, U. Lindefelt, and P. Skytt, Mater. Sci. Forum 353-356, 299 (2001).

${ }^{6}$ H. Jacobson, J. P. Bergman, C. Hallin, E. Janzén, T. Tuomi, and H. Lendenmann, J. Appl. Phys. 95, 1485 (2004).

${ }^{7}$ H. Iwata, U. Lindefelt, S. Öberg, and P. R. Briddon, Phys. Rev. B 65 , 033203 (2002).

${ }^{8}$ H. Iwata, U. Lindefelt, S. Öberg, and P. R. Briddon, J. Appl. Phys. 93, 1577 (2003).

${ }^{9}$ U. Lindefelt, H. Iwata, S. Öberg, and P. R. Briddon, Phys. Rev. B 67, 155204 (2003).

${ }^{10}$ H. P. Iwata, U. Lindefelt, S. Öberg, and P. R. Briddon, J. Appl. Phys. 94, 4972 (2003)

${ }^{11}$ For a recent review, see J. Camassel and S. Juillaguet, Phys. Status Solidi B 245, 1337 (2008).

${ }^{12}$ J. Takahashi, N. Ohtani, M. Katsuno, and S. Shinoyama, J. Cryst. Growth, 181, 229 (1997).

${ }^{13}$ J. Q. Liu, E. K. Sanchez, and M. Skowronski, Mater. Sci. Forum 389-393, 435 (2002).

${ }^{14}$ M. Syväjärvi and R. Yakimova, "Sublimation epitaxial growth of hexagonal and cubic SiC," in Encyclopedia-The Comprehensive Semiconductor Science \& Technology (SEST), edited by Pallab Bhattacharya, Roberto Fornari, and Hiroshi Kamimura (Elsevier, 2011).

${ }^{15}$ V. Jokubavicius, R. Liljedahl, Y. Y. Ou, H. Y. Ou, S. Kamiyama, R. Yakimova, and M. Syväjärvi, Mater. Sci. Forum 679-680, 103 (2011).

${ }^{16}$ M. Ikeda, H. Matsunami, and T. Tanaka, Phys. Rev. B 22, 2842 (1980).

${ }^{17}$ W. J. Choyke, D. R. Hamilton, and L. Patrick, Phys. Rev. 133, A1163 (1964).

${ }^{18}$ T. Robert, M. Marinova, S. Juillaguet, A. Henry, E. K. Polychroniadis, and J. Camassel, Mater. Sci. Forum 645-648, 347 (2010). 